\title{
Waterborne polyurethane single-ion electrolyte from aliphatic diisocyanate and various molecular length of polyethylene glycol
}

\author{
C-H. Yang ${ }^{* 1}$, W-C. Lin' ${ }^{2}$, F-J. Liu 3 \\ ${ }^{1}$ Department of Chemical and Materials Engineering, National University of Kaohsiung, Kaohsiung 811, Taiwan \\ 2Department of Environmental Engineering, Kun Shan University, Tainan 710, Taiwan \\ ${ }^{3}$ Department of Chemical Engineering, National United University, Miaoli 360, Taiwan
}

Received 3 November 2006; accepted in revised form 24 February 2007

\begin{abstract}
The waterborne polyurethane (WPU) dispersions from the reaction of cycloaliphatic diisocyanates [4,4'-methylenebis(cyclohexyl isocyanate) $\left(\mathrm{H}_{12} \mathrm{MDI}\right)$ and isophorone diisocyanate (IPDI)] and polyethylene glycol (PEG) with various molecular lengths were synthesized using our modified acetone process. Differetial scanning calorimeter (DSC) and Fourier transform infrared spectroscopy (FTIR) were utilized to characterize WPU films for the behavior of their crystallinity and H-bonding of WPU films. The $T_{g}$ value of WPU increases with increasing the molecular length of PEG, whereas the $T_{m}$ of WPU decreases with increasing PEG length. Alternating current (AC) impedance experiments were performed to determine the ionic conductivities of WPU films. The WPU gel electrolytes exhibits an ionic conductivity as high as $\sim 10^{-5} \mathrm{~S} / \mathrm{cm}$ at room temperature.
\end{abstract}

Keywords: polymer gels, waterborne polyurethane electrolyte, single ion, polyethylene glycol

\section{Introduction}

Solid polymer electrolytes have received much interest because of their use in lithium ion polymer batteries. Most of the researchers have concentrated on designing novel polymer materials possessing high ionic conductivity, good mechanical properties as well as thermal stability for practical applications [1-3]. Polyether-based electrolytes showed features such as good adherence to electrodes and the ability to solvate many inorganic salts. Ionic conductive polymers such as complexes of poly(ethylene oxide) (PEO) and lithium salts, which showed high ionic conductivity of $10^{-4}$ to $10^{-3} \mathrm{~S} / \mathrm{cm}$ at high temperature were characterized by a bi-ion transport mechanism $[4,5]$. The disadvantage of these materials is the significant decrease in ionic conductivity using direct current polarization. Therefore, an ionic conductive polymer with single-ion transport mechanism is required for a number of applications. In general, the ionic conductivity of single-ion transport materials is lower than that of the bi-ion transport materials. Several ionic conductive polymers have been characterized by a single-ion transport mechanism [6]. Most of them are polyblends of polymer and polyether or copolymers synthesized from polymers with low molecular weight of polyether. Polyblend electrolytes show poor mechanic properties, while copolymer electrolytes have low conductivity [7].

It is conceivable that ionic transport exists in the amorphous phase of polymers whereas nonconducting behavior is found in the crystalline phase [8]. Thus, to design an excellent polymer elec-

*Corresponding author, e-mail: yangch@nuk.edu.tw

(C) BME-PT and GTE 
trolyte, the crystallinity of the polymer should be decreased, as well as adequate mechanical properties should be maintained for applications. However, PEO is a semicrystalline polymer, possessing both amorphous and crystalline phases at room temperature. Hence, considerable ionic conductivity in PEO-based polymers can only be obtained at high temperature [9]. Knowing this, in designing a novel polymer of similar PEO structure, it is reasonable to react PEG with diisocyanate to form PEG-based polyurethane (PU), because PU polymer possesses high tensile strength, elasticity as well as low crystallinity. In our laboratory, a series of studies [10-14] have been performed for the polymer electrolytes on the basis of waterborne polyurethane (WPU).

Polyurethanes are composed of polyether or polyester soft segment and a diisocyanate-based hard segment, which can be characterized by a twophase morphology [13]. The phase separation is due to the fact that the hard and soft phases are immiscible and leads to the formation of a hardsegment domain, a soft-segment matrix, and an illdefined inter-phase. The hard-segment domains act as physical cross-links and filler particles to the soft segment matrix. The domain formation is derived from the intermolecular hydrogen bonding between the hard-hard segments of urethane or urea linkages. The hydrogen bonding is characterized by a frequency shift to the values lower than those corresponding to the free groups (i.e. no hydrogen bonding) in the spectrum of FTIR. Meanwhile, the extent of the frequency shift is usually used as an estimate of hydrogen-bonding strength. Particularly for polyether-based PUs, the fraction of the hydrogen-bonded carbonyls is defined by a hard-hard segment hydrogen bond $(\mathrm{N}-\mathrm{H} \cdots \mathrm{O}=\mathrm{C}$ bond $)$, which was employed to evaluate the extent of phase separation. On the other hand, the fraction of the hydrogen-bonded ether oxygen $(\mathrm{N}-\mathrm{H} \cdots \mathrm{O}-)$ represents the extent of phase mixing between hard and soft segments.

To clarify the effects of PEG molecular length and different kinds of cycloaliphatic diisocyanates on the polymer electrolyte, PEG-based WPU electrolytes were extended from the previous [12] to others by using various molecular weights of PEG as soft segments and two kinds of cycloaliphatic diisocyanates [4,4'-methylenebis(cyclohexyl isocyanate) ( $\left.\mathrm{H}_{12} \mathrm{MDI}\right)$ and isophorone diisocyanate (IPDI)] as hard segments without adding the diol chain extender DMPA (dimethyolpropionic acid). By using FTIR, DSC, and impedance spectroscopy (IS), we investigate the effects of PEG molecular weight and kinds of diisocyanates on phase variation, morphology characteristics and bulk conductivity of these WPU electrolytes.

\section{Experimental Section}

\subsection{Materials}

PEG $\left(M_{w}=1000,2000,3000\right.$ and 4000, Showa) used as the soft segment was dried at $80^{\circ} \mathrm{C}$ in a vacuum oven for $24 \mathrm{~h}$. IPDI (Lancaster) and $\mathrm{H}_{12} \mathrm{MDI}$ (Wako) were used as hard segment without further treatment. Propylene carbonate (PC) (Aldrich) was distilled at low pressure and stored over $3 \AA$ molecular sieves before use. Acetone (Tedia) was immersed in $4 \AA$ molecular sieves for more than one week before use. Di-n-butyltin(IV) dilaurate (DBTDL) (Wako), ehylenediamine (EDA) (Merck), and butane sultone (BS) (Aldrich) were used without further treatment.

\subsection{Synthesis of WPU dispersions}

Two series of WPU dispersions were prepared through our modified acetone process [1] with $\mathrm{NCO} / \mathrm{OH}=1.5$ using polyaddition of two diisocyanates $\left(\mathrm{H}_{12} \mathrm{MDI}\right.$ and IPDI) to various molecular weights of PEG. PEG was added to the reactor and heated to $50^{\circ} \mathrm{C}$. Diisocyanates and DBTDL were then added and reacted at $85^{\circ} \mathrm{C}$ under nitrogen atmosphere for $6 \mathrm{~h}$. To the final NCO-terminal PU prepolymer was added a suitable amount of acetone. The chain extender was prepared as follows [1]: Ethylenediamine $(6.0 \mathrm{~g} ; 0.1 \mathrm{~mol})$ was added drop-wise to a solution of butane sultone (13.6 g; $0.1 \mathrm{~mol})$ and $\mathrm{H}_{2} \mathrm{O}(25 \mathrm{~g})$. The resulting mixture was heated at 60 to $70^{\circ} \mathrm{C}$ for $30 \mathrm{~min}$, then cooled to room temperature, after which $\mathrm{LiOH}(1.9 \mathrm{~g}$; $0.1 \mathrm{~mol}$ ) was added. The chain extender was added immediately to the freshly prepared NCO-terminated PU prepolymer solution with the [NCO]/ $\left[\mathrm{NH}_{2}\right]$ ratio of 1.0. The ratio of $\left[\mathrm{Li}^{+}\right] /[\mathrm{EO}]$ (where EO is the concentration of ethylene oxide units in WPU) was kept at 0.005 . The resulting mixture was then heated at phase-inversion temperature of $50^{\circ} \mathrm{C}$. Doubly distilled water was added to above PU solutions at agitation rate and water addition rate of $200 \mathrm{rev} / \mathrm{min}$ and $2.0 \mathrm{ml} / \mathrm{min}$, respectively. 
An aqueous dispersion of about $30 \mathrm{wt} \%$ solids was obtained upon removal of acetone by rotary vacuum evaporation.

\subsection{Film preparation}

The films from the solvent evaporation method were obtained by casting WPU dispersion solution on a Teflon disk, followed by drying at $45^{\circ} \mathrm{C}$ for 2 days. The films were then removed to a glove box further drying. Before all tests on these films, the water content of the films was determined to be around 10 ppm using a Karl Fisher moisture titrator (MKC-210, Kyoto Electronics, Japan) for the film extracted solvent (acetone).

\subsection{Ac impedance measurement}

Impedance analysis of the polymer electrolyte was performed using a CMS300 EIS system (Gamry Instrument, USA) with SR810 DSP lock-in amplifier (Standard Research System, USA) under an oscillation potential of $10 \mathrm{mV}$ from $100 \mathrm{kHz}$ to $0.1 \mathrm{~Hz}$. The WPU films or their corresponding gel electrolytes were sandwiched by two stainless steel SS304) blocking electrodes for conductivity tests.

\subsection{DSC measurement}

Thermal analysis of WPU films was carried out using Du Pont system DSC (Du Pont 910, USA) with a temperature range from -100 to $100^{\circ} \mathrm{C}$ at a heating rate of $10^{\circ} \mathrm{C} / \mathrm{min}$ under nitrogen purging.

\subsection{FTIR spectroscopy}

The samples for infrared analysis were prepared by casting $1 \%(\mathrm{w} / \mathrm{v})$ WPU solution, acetone as solvent, directly on $\mathrm{KBr}$ pellets and drying at $120^{\circ} \mathrm{C}$ in a vacuum oven for $24 \mathrm{~h}$. Infrared spectra were obtained with a Fourier Transform IR spectrophotometer (Nicolet FTIR-550) and recorded by averaging 64 scans at a resolution of $4 \mathrm{~cm}^{-1}$.

\section{Results and discussion}

\subsection{DSC analysis}

Figure 1 shows the DSC thermograms of PEG1000, IPDI-1000 (WPU obtained from the copolymer of IPDI and PEG-1000) and $\mathrm{H}_{12} \mathrm{MDI}-1000$ (WPU obtained from the copolymer of $\mathrm{H}_{12} \mathrm{MDI}$

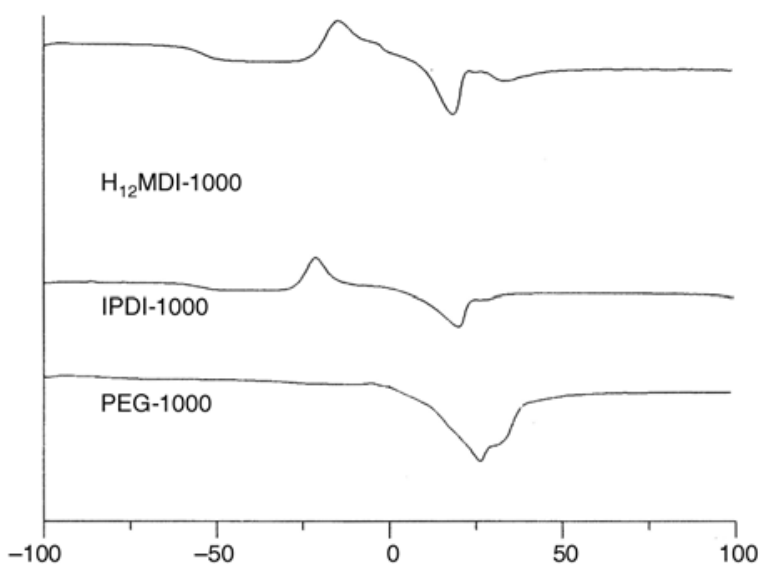

Figure 1. DSC thermograms of WPU films with PEG1000 samples at a heating rate of $10^{\circ} \mathrm{C} / \mathrm{min}$

Table 1. Phase-transition characteristics of PEG-based WPU films

\begin{tabular}{|l|c|c|c|c|}
\hline \multicolumn{1}{|c|}{ Film } & $\begin{array}{c}\mathbf{T}_{\mathbf{g}} \\
{\left[{ }^{\circ} \mathbf{C}\right]}\end{array}$ & $\begin{array}{c}\mathbf{T}_{\mathbf{m}} \\
{\left[{ }^{\circ} \mathbf{C}\right]}\end{array}$ & $\begin{array}{c}\Delta \mathbf{T} / \mathbf{S} \\
{\left[{ }^{\circ} \mathbf{C} / \mathbf{m o l}\right]}\end{array}$ & $\begin{array}{c}\Delta \mathbf{T} / \Delta \mathbf{C} \\
{\left[{ }^{\circ} \mathbf{C} / \mathbf{m g} \mathbf{g ~ W P U}\right]}\end{array}$ \\
\hline PEG-1000 & -79 & 26 & - & - \\
\hline PEG-2000 & -76 & 51 & - & - \\
\hline PEG-3000 & -72 & 58 & - & - \\
\hline PEG-4000 & -68 & 59 & - & - \\
\hline IPDI-1000 & -62 & 20 & 22.67 & 2.39 \\
\hline IPDI-2000 & -59 & 47 & 19.84 & 4.17 \\
\hline IPDI-3000 & -52 & 48 & 22.22 & 7.02 \\
\hline IPDI-4000 & -49 & 52 & 20.58 & 8.67 \\
\hline $\mathrm{H}_{12}$ MDI-1000 & -59 & 20 & 27.86 & 2.93 \\
\hline $\mathrm{H}_{12}$ MDI-2000 & -57 & 48 & 22.73 & 4.79 \\
\hline $\mathrm{H}_{12}$ MDI-3000 & -55 & 53 & 19.23 & 6.07 \\
\hline $\mathrm{H}_{12}$ MDI-4000 & -53 & 54 & 16.47 & 6.94 \\
\hline
\end{tabular}

${ }^{a}$ waterborne polyurethanes based on the copolymers of IPDI and PEG, numbers represent the molecular weight of PEG.

${ }^{b_{w}}$ aterborne polyurethanes based on the copolymers of $\mathrm{H}_{12} \mathrm{MDI}$ and PEG, numbers represent the molecular weight of PEG.

and PEG-1000) samples, indicating crystallinity for $\mathrm{H}_{12} \mathrm{MDI}-1000$ and IPDI-1000 samples as compared PEG-1000 sample. Meanwhile, a soft-segment $T_{m}$ of these samples was readily observed. A series of the soft-segment $T_{g}$ and $T_{m}$ are listed in Table 1. Note that the values of $T_{g}$ and $T_{m}$ of PEG increase with increasing molecular weight. And, the values of $T_{g}$ increase with adding IPDI and $\mathrm{H}_{12} \mathrm{MDI}$, respectively, in the WPU polymers. This reflects two effects: First, the addition of lithium salt increases $T_{g}$. Second, the segregation of between soft segment and hard segment occurred in these WPUs. For these investigated polymers, the effect of lithium salt on the polyether soft-segment $T_{g}$ is significant in the PEG-based WPUs. This indicates that the solvation of the lithium cation by the PEG soft-segment partially arrests the local motion of 
the polymer segment through the formation of transient cross-links, leading to an increase in the softsegment $T_{g}$. By normalizing the $T_{g}$ data against unit salt concentration for these WPU films, the $\Delta T_{g} / \Delta C$ was calculated at each measurement and which are listed in Table 1. It is obvious that the value of $\Delta T_{g} / \Delta C$ increases with increasing PEG molecular length in the WPUs. This result reflects that the formation of transient cross-links between lithium cation and PEG soft-segment increases with increasing the molecular weight of PEG. This arises from the higher content of ethylene oxide (EO) per gram of WPU with the higher molecular weight of PEG. Furthermore, the specific variation of $T_{g}$ is defined as $\Delta T_{g} / S$, where $S$ is the soft content, and is also listed in Table 1. It is interesting that these values remain around 20.0 for IPDIbased WPUs, but the value decreases with increasing the length of PEG molecules for $\mathrm{H}_{12} \mathrm{MDI}$-based WPUs. This may be because the strength of Hbonding decreases with increasing PEG length in $\mathrm{H}_{12}$ MDI-based WPUs (see below). In contrast, the values of $T_{m}$ decrease with adding IPDI and $\mathrm{H}_{12} \mathrm{MDI}$, respectively, in WPUs. These results indicate that the polymers have a certain degree of hard- and soft-segment mixing. The decrease of soft-segment $T_{m}$ values for these investigated polymers was observed in the range of $5-10^{\circ} \mathrm{C}$. In comparison with both of $T_{g}$ increase and $T_{m}$ decrease of these WPU polymers, it is obvious that the segregation of between soft- and hard-segments is more significant than the hard- and soft-segment mixing. This result is very different from that with the addition of DMPA content in WPU polymers [12]; the latter implies that the degree of mixing of hard- and soft- segments can be enhanced by the addition of DMPA. In addition, note in Figure 1 that the devitrification exists in both IPDI- and $\mathrm{H}_{12} \mathrm{MDI}$-based WPUs, but occurring at exotherm and is followed by an endotherm at a higher temperature that corresponds to the melting of these crystals (IPDI- and $\mathrm{H}_{12} \mathrm{MDI}$-based WPUs). This suggests that there should exist a nucleating phase of crystal structure in these WPU polymers during temperature scanning. After nucleation, crystal growth rates are small because the glass phase is heated to a higher temperature, at which crystal growth occurs on the surface of the existing nuclei. Because there is a large number of nuclei present, distributed throughout the bulk of the glass, each can grow by only a small amount until it impringes on neighboring nuclei. It is noteworthy that the devitrification cannot be observed in both of pure PEG and high DMPA-introducing WPUs [12]. Moreover, the devitrification temperature of $\mathrm{H}_{12}$ MDI-based WPUs is generally higher than that of IPDI-based WPUs; this result could be related to the H-bonding between hard-hard and hard-soft segments.

\subsection{Evaluation of FTIR spectra}

In this study, the addition of different PEG molecular weight was achieved by keeping constant $\mathrm{NCO} / \mathrm{OH}$ ratio of 1.5 in the WPU chain. Therefore, the content of lithium salt $(\mathrm{LiOH})$ decreases with increasing the molecular weight of PEG because the chain extension was completed with the residual $\mathrm{NCO} / \mathrm{NH}_{2}$ ratio of 1.0. A typical FTIR spectrum is shown in Figure 2. Four main regions are presented: (i) $3000-3600 \mathrm{~cm}^{-1} \mathrm{~N}-\mathrm{H}$ stretching, a broad absorption band of the $\mathrm{N}-\mathrm{H}$ region was observed; (ii) $1600-1750 \mathrm{~cm}^{-1} \mathrm{C}=\mathrm{O}$ stretching, the carbonyl stretching absorption band was observed as two peaks. The higher frequency at around $1700 \mathrm{~cm}^{-1}$ was assigned to free (i.e., not H-bonded) carbonyl stretching whilst the peak at around $1600 \mathrm{~cm}^{-1}$ was attributed to H-bonded carbonyl stretching; (iii) $1500-1600 \mathrm{~cm}^{-1}$ (-COO) stretching, characteristic of the urethane group; (iv) $1000-1150 \mathrm{~cm}^{-1} \mathrm{C}-\mathrm{O}-\mathrm{C}$ stretching, the ether oxygen of the soft segment.

FTIR spectra have demonstrated H-bonding in PU $[12,15]$; the strength of the H-bonding was measured by frequency shifts to values lower than those observed when these groups are free. The $\mathrm{N}-\mathrm{H}$ group could form hard-hard segment H-bonding with the carbonyl oxygen and hard-soft segment

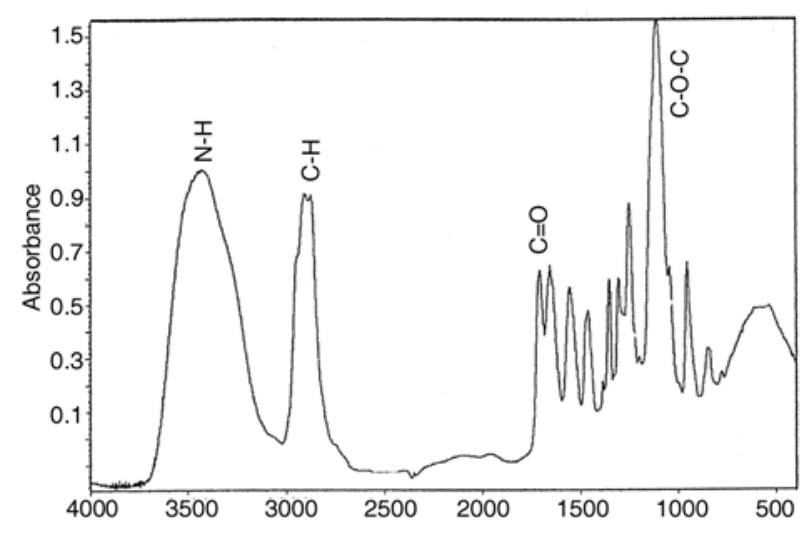

Figure 2. Typical FTIR spectrum of IPDI-1000 WPU film 
$\mathrm{H}$-bonding with the ether oxygen. In general, the strength of hard-hard segment $\mathrm{H}$-bonding is stronger than that of hard-soft segment H-bonding [7]. Deconvolution of the $\mathrm{N}-\mathrm{H}$ stretching region was done and the best fits were obtained using a Gaussian-Lorentzian sum as seen in Figure 3. This indicates that the higher frequency of the deconvoluted peak (peak 3 ) is assigned to free $\mathrm{N}-\mathrm{H}$ stretching (about $3550 \mathrm{~cm}^{-1}$ ), lower frequency (peak 1) is assigned to $\mathrm{N}-\mathrm{H}$ stretching which is affected by hard-hard segment H-bonding (about $3300 \mathrm{~cm}^{-1}$ ). The central peak (peak 2) is $\mathrm{N}-\mathrm{H}$ stretching which is affected by hard-soft segment H-bonding (about $3430 \mathrm{~cm}^{-1}$ ). The frequency shift of $\mathrm{H}$-bonded $\mathrm{N}-\mathrm{H}$ stretching represents the stretching of $\mathrm{H}$-bonding in polyurethane $[13,16]$. The area of each band was determined by using the Nelder-Mead optimization method. All $\mathrm{N}-\mathrm{H}$ band areas were normalized on the basis of total $\mathrm{N}-\mathrm{H}$ stretching band area and are

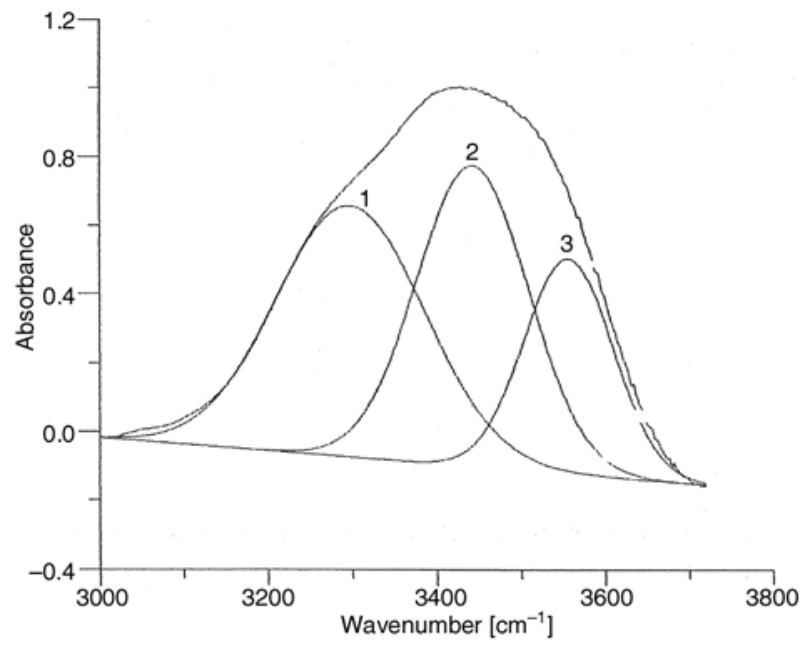

Figure 3. FTIR spectrum and deconvolution curves of IPDI-1000 WPU film in $\mathrm{N}-\mathrm{H}$ stretching region

Table 2. Decomposition results of WPUs in the $\mathrm{N}-\mathrm{H}$ stretching region

\begin{tabular}{|l|c|c|c|c|c|c|}
\hline \multirow{3}{*}{ Film } & \multicolumn{3}{|c|}{$\begin{array}{c}\text { Frequency shift } \\
{\left[\mathbf{c m}^{-1} \text { ] }\right.}\end{array}$} & \multicolumn{3}{c|}{$\begin{array}{c}\text { Peak area } \\
{[\%]}\end{array}$} \\
\cline { 2 - 8 } & H-H $^{*}$ & H-S $^{* *}$ & $\begin{array}{c}\text { Free N-H } \\
\text { stretching }\end{array}$ & $\mathbf{1}$ & $\mathbf{2}$ & $\mathbf{3}$ \\
\hline $\mathrm{H}_{12}$ MDI-1000 & 280 & 160 & 20 & 23.20 & 45.10 & 31.70 \\
\hline $\mathrm{H}_{12}$ MDI-2000 & 268 & 151 & 16 & 26.51 & 37.43 & 33.05 \\
\hline $\mathrm{H}_{12}$ MDI-3000 & 257 & 143 & 11 & 30.05 & 37.10 & 32.85 \\
\hline $\mathrm{H}_{12}$ MDI-4000 & 233 & 113 & 8 & 31.75 & 34.24 & 34.01 \\
\hline IPDI-1000 & 260 & 133 & 25 & 25.01 & 42.86 & 32.13 \\
\hline IPDI-2000 & 240 & 127 & 21 & 27.85 & 39.01 & 33.14 \\
\hline IPDI-3000 & 233 & 120 & 17 & 30.45 & 35.54 & 34.01 \\
\hline IPDI-4000 & 227 & 112 & 12 & 33.12 & 32.92 & 33.96 \\
\hline
\end{tabular}

*Hard-hard segment H-bonded N-H stretching.

**Hard-soft segment H-bonded $\mathrm{N}-\mathrm{H}$ stretching.

aThe band areas are based on the total $\mathrm{N}-\mathrm{H}$ stretching area. listed in Table 2, revealing that the band shift of three decomposition peaks is almost proportional to the lithium salt concentration. The peak position of free $\mathrm{N}-\mathrm{H}$ stretching (peak 3 ) with salt addition is higher than that peak position $\left(3450 \mathrm{~cm}^{-1}\right)$ without the salt doping. This is due to the interaction between the $\mathrm{Li}^{+1}$ cation and the lone pair of electrons on nitrogen atom [14], leading to the reduction of $\mathrm{N}-\mathrm{H}$ bond length. Thus, the vibration energy of $\mathrm{N}-\mathrm{H}$ bond is relatively increased when the salt is doped in WPU film, resulting in a higher frequency of absorption peak. Table 2 shows that the area ratio of peak 3 is insignificantly influenced by the concentration of doping salt for both of IPDI- and $\mathrm{H}_{12} \mathrm{MDI}-$ based WPUs.

In Table 2, the component of peak 1 corresponds to hydrogen bonding between $\mathrm{N}-\mathrm{H}$ and carbonyl groups [14]. The position of this component shifts to higher frequency as the salt content increases for both of the two WPU systems. Because band position is related to the strength of $\mathrm{H}$-bonded $\mathrm{N}-\mathrm{H}$ bond, the shift to higher frequency with high salt concentration indicates a decrease in the H-bonded strength. Therefore, the H-bonding strength is decreased with increasing salt content. This is possibly due to the localization of the electron-rich oxygen atoms on the carbonyl through coordination of the $\mathrm{Li}^{+1}$ cation with the $\mathrm{H}$-bonded species. Furthermore, the band area of peak 1 decreased with increasing salt content in Table 2 . This reflects that the possibility of the hydrogen bonding between $\mathrm{N}-\mathrm{H}$ and carbonyls is decreased.

Note in Table 2 reveals the band position of hydrogen bonding of $\mathrm{N}-\mathrm{H}$ to $-\mathrm{O}-$ (ether oxygen) (peak 2) [14] of PEG soft segment is also shifted to higher frequency with the addition of salt. This shift of frequency to higher values increases with increasing salt content, implying that the more added salt in WPU gives the stronger band strength of $\mathrm{N}-\mathrm{H}$ bond. This is likely due to the coordination of nonbonded electrons on the ether oxygen atoms with the $\mathrm{Li}^{+1}$ cation, leading to a weakening of the hydrogen-bonded strength between $\mathrm{N}-\mathrm{H}$ and ether oxygen atoms. An examination of Table 2 reveals that the band area of $\mathrm{NH}$-ether (peak 2) increases with increasing salt content. This can be attributed to the fact that the coordination of non-bonded electrons on ether oxygen atoms with cation is increased, inducing an increase in hydrogen bonding between $\mathrm{N}-\mathrm{H}$ and ether groups. 
On the other hand, the strength of H-bonding stretching of $\mathrm{H}_{12} \mathrm{MDI}$-based WPUs is higher than that of IPDI-based WPUs for both hard-hard and hard-soft segments. This implies that the molecular architectures of $\mathrm{H}_{12}$ MDI-based WPUs are beneficial to form the H-bonding between hard-hard and hard-soft segments as compared with IPDI-based WPUs. This observation may be related to the result of devitrification temperatures.

\subsection{Ionic conductivity}

Since these WPU samples possess different molecular weight of PEG in the polymer chains, it is interesting to investigate the ionic conducting behavior of these samples. Thus, AC impedance was employed to determine the conductivity $(\sigma)$ of these films. The result was plotted as a Cole-Cole plot to show the real/image parts of impedance at

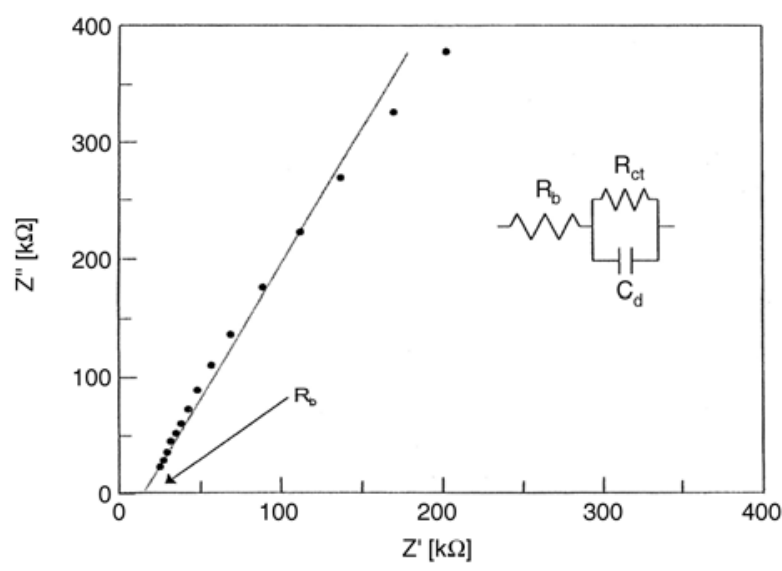

Figure 4. Cole-Cole plot of SS/WPU film (IPDI-2000)/SS at $25^{\circ} \mathrm{C} ; 150 \mu \mathrm{m}$ thickness and $0.785 \mathrm{~cm}^{2}$ area for the film. Impedance frequency from $100 \mathrm{kHz}$ to $100 \mathrm{~Hz}$. SS represents the stainless steel. Insert is the equivalent circuit

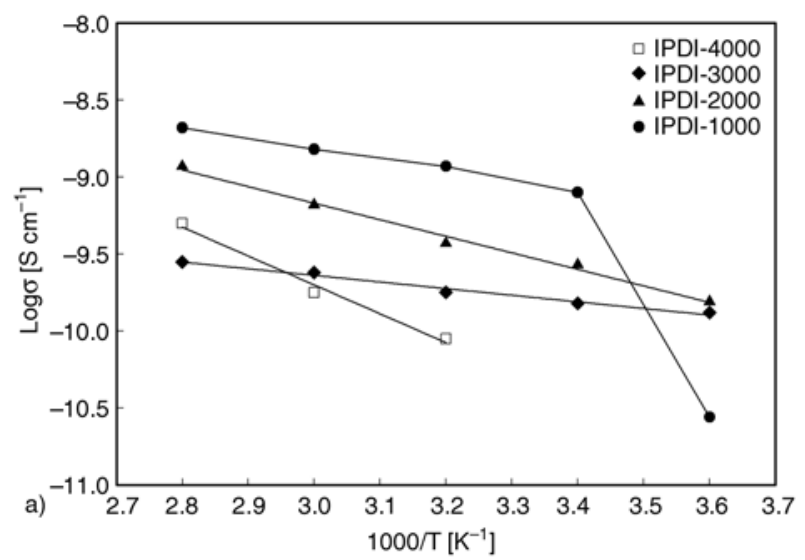

various frequencies. A typical Cole-Cole plot of WPU film sandwiched between stainless steel electrodes is shown in Figure 4. The profile shows a straight line in which the impedance decreases with increasing frequency. This indicates that the interfacial impedance decreases with increasing frequency, which is attributed to double layer formation and charge transfer reaction. At a frequency approaching $100 \mathrm{kHz}$, the line closes to the real axis, indicating that the impedance approaches pure resistance. In this case, the interfacial impedance is negligible and the bulk resistance $\left(R_{b}\right)$ is obtained. The conductivity of WPU film can be determined by letting $\sigma=\left(1 / R_{b}\right)(L / A)$, where $L$ and A represent film thickness and surface area of the WPU film, respectively. Figure 5 shows the Arrhenius plot of ionic conductivity for WPU films. It is obvious that IPDI-PEG (Figure 5a) and $\mathrm{H}_{12}$ MDI-PEG series (Figure 5b) have similar behavior of conductivity. The conductivity of these WPU films increases with decreasing the molecular weight of PEG. This is ascribable to the more significant hard-and soft-segment mixing exists in PEG-1000 WPUs as compared with other samples, resulting in the formation of amorphous state in WPU polymers which is readily to transport $\mathrm{Li}^{+}$ion in the polymer matrix. It also indicates that the conductivities of these samples almost obey the Arrhenius law but PEG-1000 sample possesses a transition point around $20^{\circ} \mathrm{C}$. This transition point reflects the fact that this polymer has $T_{m}=20^{\circ} \mathrm{C}$ (see Table 1), as the migration of lithium ion in polymer electrolytes should be easier in melted (amorphous) polymer state than in the crystalline state.

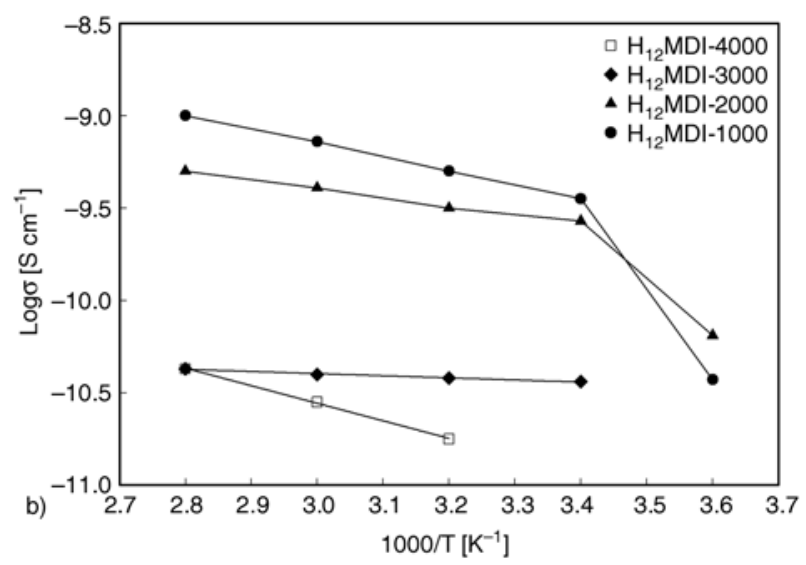

Figure 5. Arrhenius plot of conductivity for WPU films with various molecular weights of PEG. (a) IPDI and (b) $\mathrm{H}_{12} \mathrm{MDI}$ samples 

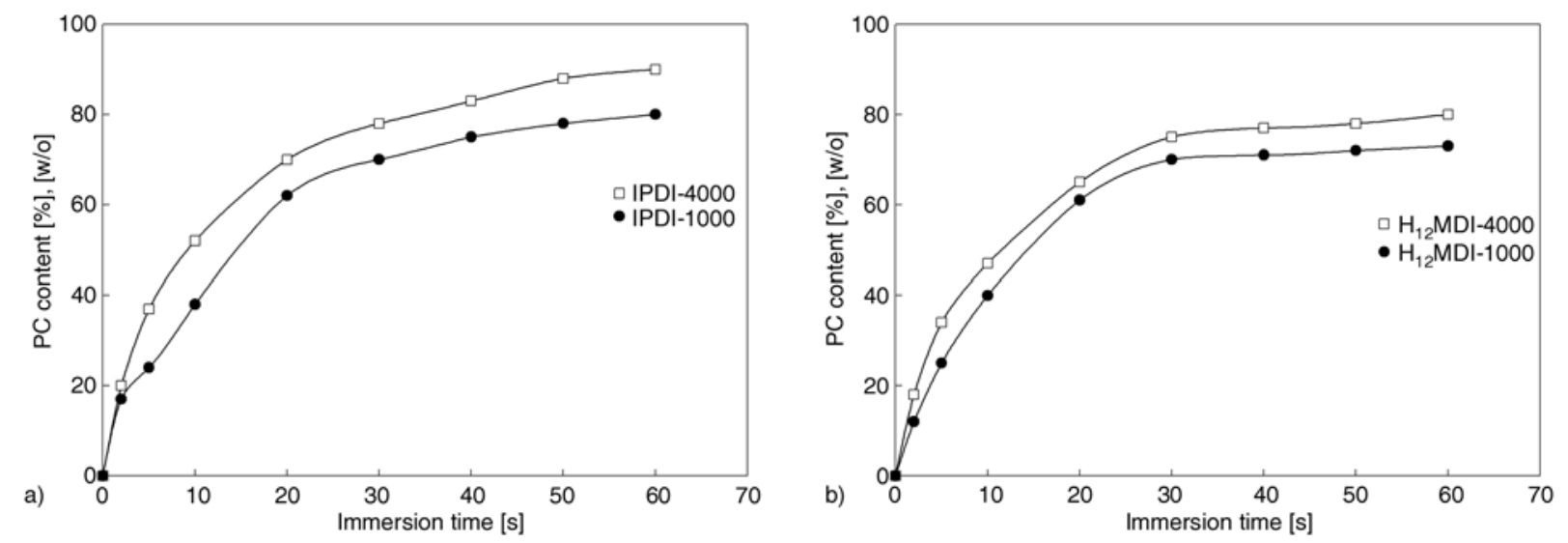

Figure 6. PC swelling rate of WPU films with various molecular weights of PEG. (a) IPDI and (b) $\mathrm{H}_{12}$ MDI samples

In order to improve the conductivity, these WPU samples were impregnated with PC to form geltype electrolytes. The swollen weight was measured with various immersion times and the results were plotted in Figure 6. It is clear that the curves are saturated for all of IPDI and $\mathrm{H}_{12} \mathrm{MDI}$ samples. The swelling rate may be obtained from the slope of the curves; the saturated soaking of solvent (PC) can be attained after $1 \mathrm{~min}$ immersion of the WPU films. Note that the swelling rate and the saturated swollen quantity increase with increasing molecular weight of PEG. This reflects that the shorter soft segment (PEG) in WPU yields the more contents of urethane groups. This readily forms the $\mathrm{H}$-bonding between hard-hard and hard-soft segments to retard PC uptaking, and thus PC is mainly absorbed in soft phase of PEG. To understand the effects of PEG molecular weight on the conductivity of WPU gel electrolytes, the swollen PC in these WPU samples was controlled at about $50 \mathrm{wt} \%$ and consequently AC impedance was performed. The conductivity data as shown in Figure 7 was ana-

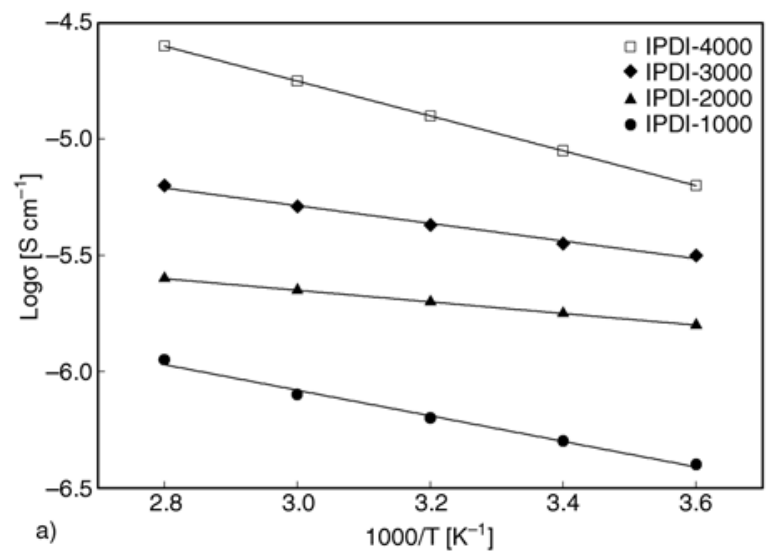

lyzed using the Arrhenius phenomenological relationship as follows (1):

$$
\sigma(T)=A \exp ^{-\frac{E}{k_{b} T}}
$$

The Arrhenius formula is used when the ions are decoupled from the polymer host and activated hopping is required for ionic transport. In Figure 7, these straight lines imply that the conductivity of these films almost obeys Arrhenius law, and therefore, the activated hopping predominates in the conductivity process. The conductivity of gel-type WPU films (Figure 7) is higher than that of the solvent-free films as compared with the results of Figure 6. Also, there is no transition point in PEG1000 WPU samples. This is ascribable that the lithium ion is completely solvated by PC solvent, resulting in the collapse of crystalline and H-bonding structures in these samples. Hence, the ion-conduction behavior of the gel film is quite different from that of the solvent-free film. Moreover, the conductivity increases with increasing molecular

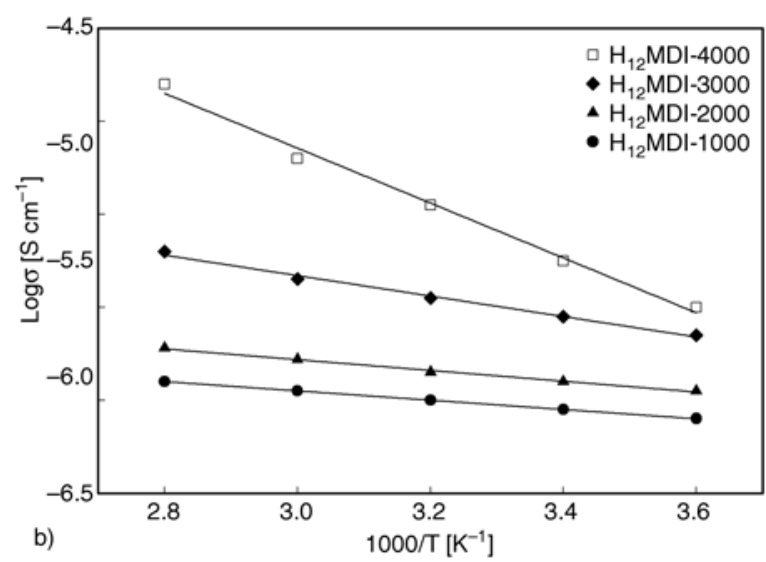

Figure 7. Arrhenius plot of conductivity for WPU gel electrolytes (containing $50 \mathrm{wt} \% \mathrm{PC}$ ) with various molecular weights of PEG. (a) IPDI and (b) $\mathrm{H}_{12}$ MDI samples 
weight of PEG in gel electrolytes, indicating that the increase in the flexibility of polymer would promote the conductivity due to more channels for the mobility of $\mathrm{Li}^{+}$ions. Without adding the diol chain extender DMPA in these WPUs, a suitable choice of soft segment in the WPUs is possibly used as a single-ion electrolyte having a similar $\sim 10^{-(4-5)} \mathrm{S} / \mathrm{cm}$ at room temperature.

\section{Conclusions}

The $T_{g}$ increases with the introduction of cycloaliphatic diisocyanates into PEG forming WPUs; the strength of H-bonding between hardhard and hard-soft segments decreases with increasing molecular weight of PEG in WPUs. The molecular architectures of $\mathrm{H}_{12} \mathrm{MDI}$-based WPUs are beneficial to form the H-bonding between hardhard and hard-soft segments as compared with IPDI-based WPUs. The hard-soft segment mixing obviously exists in the IPDI- and $\mathrm{H}_{12}$ MDI-1000 samples. Without adding DMPA, WPUs with the more significant phase separation possess the lower conductivity as single-ion electrolytes in IPDI- and $\mathrm{H}_{12} \mathrm{MDI}-2000$, 3000, 4000 samples. The conductivity of WPU gel electrolytes increases with increasing molecular weight of PEG. Thus, a WPU gel electrolyte of PEG-4000 exhibits an ionic conductivity as high as $\sim 10^{-(4-5)} \mathrm{S} / \mathrm{cm}$ at room temperature without adding the diol chain extender DMPA in these WPUs,. This demonstrates that this type of WPU gel electrolyte has potential for use as a single-ion electrolyte.

\section{Acknowlegements}

The financial support of this work by the National Science Council of the Republic of China under contract NSC 952221-E-218-060, is gratefully acknowledged.

\section{References}

[1] Yang C. H., Lin S. M., Wen T. C.: Application of statistical experimental strategies to the process optimization of waterborne polyurethane. Polymer Engineering and Science, 35, 722-730 (1995).

[2] Murata K.: An overview of research and development of solid polymer electrolyte batteries. Electrochimica Acta, 40, 2177-2184 (1995).

[3] Fauteux D., Massucco A., McLin M., Van Buren M., Shi J.: Lithium polymer electrolyte rechargeable battery. Electrochimica Acta, 40, 2185-2190 (1995).
[4] Barthel J., Buestrich R., Carl E., Gores H. J.: A new class of electrochemically and thermally stable lithium salts for lithium battery electrolytes. Journal of the Electrochemical Society, 143, 3565-3571 (1996).

[5] Ramanujachary K. V., Tong X., Lu Y., Kohn J., Greenblatt M.: Lithium ionic conductivity in poly(ether urethanes) derived from poly(ethylene glycol) and lysine ethyl ester. Journal of Applied Polymer Science, 63, 1449-1456 (1997).

[6] Xu H. S., Yang C. Z.: The ionic conductive property of sulfonated polyethylene oxide polyurethane ionomers. Journal of Polymer Science Part B-Polymer Physics, 33, 745-751 (1995).

[7] Petrovic Z. S., Javni I.: The effect of soft-segment length and concentration on phase separation in segmented polyurethanes. Journal of Polymer Science Part B-Polymer Physics, 27, 545-560 (1989).

[8] Berthier C., Gorecki W., Minier M., Armand M. B., Chabagno J. M., Rigand P.: Microscopic investigation of ionic conductivity in alkali metal salts-poly(ethylene oxide)adducts. Solid State Ionics, 11, 91-95 (1983).

[9] Gadjourova Z., Andreev Y. G., Tunstall D. P., Bruce P. G.: Ionic conductivity in crystalline polymer electrolytes. Nature, 412, 520-523 (2001).

[10] Cheng T. T., Wen T. C.: Novel water-borne polyurethane based electrolytes for lithium batteries-(I) tailor-made polymer. Journal of Electroanalytical Chemistry, 459, 99-110 (1998).

[11] Cheng T. T., Wen T. C.: Novel waterborne polyurethane based electrolytes for lithium batteries-(II) the effect of adding $\mathrm{LiCF}_{3} \mathrm{SO}_{3}$-PC. Solid State Ionics, 107, 161-171 (1998).

[12] Wen T. C., Wang Y. J., Cheng T. T., Yang C. H.: The effect of DMPA units on ionic conductivity of PEGDMPA-IPDI waterborne polyurethane as single-ion electrolytes. Polymer, 40, 3979-3988 (1999).

[13] Wen T. C., Wu M. S., Yang C. H.: Spectroscopic investigations of poly(oxypropylene) glycol-based waterborne polyurethane doped with lithium perchlorate. Macromolecules, 32, 2712-2720 (1999).

[14] Wen T. C., Luo S. S., Yang C. H.: Ionic conductivity of polymer electrolytes derived from various diisocyanate-based waterborne polyurethanes. Polymer, 41, 6755-6764 (2000).

[15] Van Heumen J. D., Stevens J. R.: The role of lithium salts in the conductivity and phase morphology of a thermoplastic polyurethane. Macromolecules, 28, 4268-4277 (1995).

[16] Teo L-S., Chen C-Y., Kuo J-F.: Fourier transform infrared spectroscopy study on effects of temperature on hydrogen-bonding in amine-containing polyurethanes and poly(urethane-urea)s. Macromolecules, 30, 1793-1799 (1997). 